\title{
Contralateral acute vascular occlusion following revascularization surgery for moyamoya disease
}

\author{
Eric S. Sussman, MD, Venkatesh Madhugiri, MCh, Mario Teo, MD, Troels H. Nielsen, MD, PhD, \\ Sunil V. Furtado, MD, Arjun V. Pendharkar, MD, Allen L. Ho, MD, Rogelio Esparza, MD, \\ Tej D. Azad, MS, Michael Zhang, MD, and Gary K. Steinberg, MD, PhD
}

Department of Neurosurgery and Stanford Stroke Center, Stanford University School of Medicine, Stanford, California

\begin{abstract}
OBJECTIVE Revascularization surgery is a safe and effective surgical treatment for symptomatic moyamoya disease (MMD) and has been shown to reduce the frequency of future ischemic events and improve quality of life in affected patients. The authors sought to investigate the occurrence of acute perioperative occlusion of the contralateral internal carotid artery (ICA) with contralateral stroke following revascularization surgery, a rare complication that has not been previously reported.
\end{abstract}

METHODS This study is a retrospective review of a prospective database of a single surgeon's series of revascularization operations in patients with MMD. From 1991 to 2016, 1446 bypasses were performed in 905 patients, 89.6\% of which involved direct anastomosis of the superficial temporal artery (STA) to a distal branch of the middle cerebral artery (MCA). Demographic, surgical, and radiographic data were collected prospectively in all treated patients.

RESULTS Symptomatic contralateral hemispheric infarcts occurred during the postoperative period in 34 cases (2.4\%). Digital subtraction angiography (DSA) was performed in each of these patients. In 8 cases $(0.6 \%)$, DSA during the immediate postoperative period revealed associated new occlusion of the contralateral ICA. In each of these cases, revascularization surgery involved direct anastomosis of the STA to an $\mathrm{M}_{4}$ branch of the MCA. Preoperative DSA revealed moderate $(n=1)$ or severe $(n=3)$ stenosis or occlusion $(n=4)$ of the ipsilateral ICA and mild $(n=2)$, moderate $(n=4)$, or severe $(n=2)$ stenosis of the contralateral ICA. The baseline Suzuki stage was $4(n=7)$ or $5(n=1)$. The collateral supply originated exclusively from the intracranial circulation in $4 / 8$ patients $(50 \%)$, and from both the intracranial and extracranial circulation in the remaining $50 \%$ of patients. Seven (88\%) of 8 patients improved symptomatically during the acute postoperative period with induced hypertension. The modified Rankin Scale (mRS) score at discharge was worse than baseline in $7 / 8$ patients $(88 \%)$, whereas 1 patient had only minor deficits that did not affect the mRS score. At the 3-year follow-up, $3 / 8$ patients $(38 \%)$ were at their baseline mRS score or better, 1 patient had significant disability compared with preoperatively, 2 patients had died, and 1 patient was lost to follow-up. Three-year follow-up is not yet available in 1 patient.

CONCLUSIONS Acute occlusion of the ICA on the contralateral side from an STA-MCA bypass is a rare, but potentially serious, complication of revascularization surgery for MMD. It highlights the importance of the hemodynamic interrelationships that exist between the two hemispheres, a concept that has been previously underappreciated. Induced hypertension during the acute period may provide adequate cerebral blood flow via developing collateral vessels, and good outcomes may be achieved with aggressive supportive management and expedited contralateral revascularization. https://thejns.org/doi/abs/10.3171/2018.8.JNS18951

KEYWORDS moyamoya disease; revascularization surgery; contralateral acute vascular occlusion; internal carotid artery; ICA; vascular disorders

$\mathrm{M}$ OYAMOYa disease (MMD) is a progressive, idiopathic steno-occlusive disease of the cerebrovasculature, which most commonly affects the supraclinoid internal carotid arteries (ICAs) as well as the proximal portions of the anterior and middle cerebral arteries. $^{20,21}$ Natural history studies have demonstrated relentless disease progression in nonsurgically managed patients, with development of ischemic stroke in as many as $82 \%$ of patients within 5 years of symptom onset. ${ }^{3,8,10}$, $14,15,19,20$ Surgical revascularization has been repeatedly

ABBREVIATIONS BP = blood pressure; $D S A=$ digital subtraction angiography; $D W I=$ diffusion-weighted imaging; ICA = internal carotid artery; $M C A=$ middle cerebral artery; MMD = moyamoya disease; $\mathrm{mRS}=$ modified Rankin Scale; $\mathrm{POD}=$ postoperative day; $\mathrm{STA}=$ superficial temporal artery; TIA = transient ischemic attack. SUBMITTED April 16, 2018. ACCEPTED August 3, 2018.

INCLUDE WHEN CITING Published online December 14, 2018; DOI: 10.3171/2018.8.JNS18951. 
shown in large, prospective case series to decrease the risk of subsequent stroke and transient ischemic attacks (TIAs), and to improve quality of life..$^{3,4,7,17,19}$ Despite the long-term benefit of surgical revascularization, however, there is an initial increase in stroke risk during the perioperative period. $4,7,19$

The reason for this relative increase in stroke risk is likely multifactorial. At baseline, MMD patients have a tenuous cerebral blood supply characterized by decrease or loss of hemodynamic reserve, autoregulatory dysfunction secondary to chronic hypoperfusion, and fragile collateral vessels. Thus, these patients may be unable to compensate for routine hemodynamic fluctuations that inevitably occur in the perioperative period. Further, when revascularization is achieved with a superficial temporal artery to middle cerebral artery (STA-MCA) bypass, the direct manipulation of an already tenuous cerebral perfusion pattern may result in a devastating alteration of cerebral blood flow dynamics.

Reports of previous case series have described cases with rapid progression of intracranial stenoses following STA-MCA bypass for atherosclerotic arterial disease, with some patients progressing to acute occlusion of an already stenotic lesion ipsilateral to the bypass in the immediate postoperative period. ${ }^{2,5,6}$ The proposed etiology of this phenomenon is decreased velocity, or perhaps even reversal of the direction, of blood flow across a preexisting stenosis, thereby resulting in stasis and subsequent occlusion. ${ }^{16}$ More rarely, the contralateral ICA may become occluded in the immediate postoperative period following STA-MCA bypass. To our knowledge, this phenomenon has not been previously reported in the literature. Here, we report on 8 cases of acute contralateral ICA occlusion following revascularization surgery for MMD.

\section{Methods}

This was an institutional review board (IRB)-approved retrospective review of a single surgeon's prospectively maintained database of MMD patients at Stanford University Medical Center. The database was queried to identify all patients with transient or persistent new neurological deficits following STA-MCA bypass. For each of these patients, the pre- and postoperative MR images were reviewed to identify new diffusion-weighted imaging (DWI)-positive lesions associated with the clinical findings. This subgroup of patients with new DWI-positive lesions was further stratified according to the side of the diffusion restriction relative to the STA-MCA bypass. In patients with new DWI-positive lesions in the contralateral hemisphere from the revascularization procedure, the preand postoperative digital subtraction angiography (DSA) images were carefully reviewed to identify the cohort of patients with acute contralateral ICA occlusion.

Demographic, clinical, and radiographic data were reviewed in detail for each of the patients found to have acute contralateral ICA occlusion following STA-MCA bypass. This included review of continuous flowsheets of vital signs, medication administration records, and laboratory values, as well as pre- and postoperative head CT scans, MR images, and DSA studies. ICA stenosis on DSA was classified as mild ( $<50 \%$ stenosis), moderate (50\%-90\% stenosis), severe ( $>90 \%$ stenosis), or occluded.

The primary clinical outcome measure was the modified Rankin Scale (mRS) score, which was obtained from follow-up clinic visits and electronic communication (emails and telephone calls). Patients also underwent standardized serial neuroimaging studies (MRI, DSA, and cerebrovascular reserve studies) at 6 months and 3 years postoperatively. Clinical and radiographic follow-up data were recorded prospectively in the MMD database.

\section{Results}

From 1991 to 2016, the senior author (G.K.S.) performed 1446 bypasses in 905 patients with MMD. Direct STA-MCA anastomosis was performed in $89.6 \%$ of these operations. The surgical technique, including the neuroanesthetic, was standardized across all operations, and has been previously described. ${ }^{16}$

New DWI-positive lesions were identified on postoperative MRI (performed 0-1 day following surgery) in 122 cases $(8.4 \%)$. These DWI-positive lesions were associated with new clinical deficits in 80 cases $(5.5 \%)$, and the symptomatic DWI-positive lesion was located on the side contralateral to the bypass in 34 cases $(2.4 \%)$. Eight cases $(0.6 \%)$ were associated with perioperative occlusion of the contralateral ICA.

Among this cohort of 8 patients with acute contralateral ICA occlusion, 6 (75\%) were female, and 7 (88\%) were Caucasian (1 patient was Asian). The mean age at the time of surgery was 37.9 years (range 22-51 years). Hypercoagulability workup was performed in $3 / 8$ patients (38\%); 2 of these patients were heterozygous for a factor $\mathrm{V}$ Leiden mutation, and the third patient tested positive for antiphospholipid antibodies. The remainder of the hypercoagulability workup was otherwise negative in these 3 patients.

The baseline median mRS score was 2 (range 1-3). Preoperative MRI (performed 1-7 days prior to surgery) demonstrated bilateral FLAIR hyperintensities in 7 patients $(88 \%)$; 2 patients $(25 \%)$ also had DWI-positive lesions (ipsilateral to the bypassed hemisphere in one patient, and bilateral in the other patient). One patient had no radiographic evidence of ischemic injury on preoperative MRI. Preoperative MR-perfusion imaging revealed no evidence of contralateral hemisphere perfusion delay in $3 / 8$ patients $(38 \%)$, contralateral perfusion delay with appropriate Diamox-induced augmentation in $3 / 8$ patients (38\%), and contralateral hemisphere perfusion delay with inappropriate Diamox-induced augmentation in $2 / 8$ patients $(25 \%)$. Preoperative DSA was performed no more than 7 days prior to revascularization in $7 / 8$ patients $(88 \%)$. One patient underwent preoperative DSA approximately 3 months prior to surgical revascularization. Bilateral disease was present at the time of initial presentation in all patients. The collateral vascular network was purely intracranial (i.e., from the vertebrobasilar circulation, contralateral ICA, or lenticulostriate "moyamoya" vessels) in 4 patients $(50 \%)$, while intracranial and extracranial (i.e., from the external carotid artery circulation) collaterals were present in the remaining $50 \%$ of patients. Preoperative DSA revealed severe stenosis (4 patients) or occlusion 
(4 patients) of the ipsilateral ICA, and mild ( 2 patients), moderate (4 patients), or severe (2 patients) stenosis of the contralateral ICA. In the cohort of patients with postoperative symptomatic contralateral infarcts without acute occlusion of the contralateral ICA $(n=26)$, the preoperative status of the contralateral ICA was occluded in 15/26 patients $(58 \%)$ and severely stenotic in 11/26 (42\%), while the ipsilateral ICA was occluded in $16 / 26$ patients $(62 \%)$, severely stenotic in $7 / 26$ patients $(27 \%)$, and mildly stenotic in $3 / 26$ patients $(12 \%)$.

Direct anastomosis of the STA to an $\mathrm{M}_{4}$ branch of the MCA was performed in all 8 patients. Anesthesia records did not reveal any unusual intraoperative hemodynamic events or fluctuations, with mean arterial pressures typically maintained between 80 and $100 \mathrm{~mm} \mathrm{Hg}$ for the duration of the revascularization procedure. All patients were noted to be at their preoperative neurological baselines upon initial recovery from anesthesia. Symptom onset occurred within 12 hours of completion of surgical revascularization in $8 / 8$ patients $(100 \%)$. MRI with perfusion imaging was obtained urgently upon symptom onset and revealed exacerbation of contralateral perfusion deficit in $8 / 8$ patients $(100 \%)$, as well as new contralateral DWI hyperintensity in $7 / 8$ patients $(88 \%)$. Perfusion imaging in the ipsilateral hemisphere demonstrated stable (4 patients) or increased (4 patients) size of the region of perfusion delay in $8 / 8$ patients $(100 \%)$, with the exception of a small territory of improvement at the site of the direct bypass graft in $3 / 8$ patients $(38 \%)$. The 1 patient without contralateral DWI hyperintensity on the initial MRI developed contralateral DWI-positive lesions by the time of repeat MRI on postoperative day (POD) 5. Subsequent DSA, obtained on POD 1 in 7/8 patients and on POD 2 in 1/8 patients, revealed interval progression to complete occlusion of the contralateral ICA, compared with preoperative DSA. Radiographic images from 2 representative patients are provided in Figs. 1 and 2.

During the acute period, all patients were treated in the critical care setting with induced hypertension. Symptom severity was blood pressure (BP) dependent in $7 / 8$ patients (88\%); 1 patient (patient 4) had severe neurological deficits immediately upon symptom onset that did not improve with BP augmentation. Five patients $(63 \%)$ underwent direct STA-MCA bypass of the contralateral hemisphere during the same hospital stay (patients 1-3, 6, and 7), and no new DWI infarcts were noted on subsequent MR images in any of these 5 patients. Among the 3 patients who did not undergo contralateral revascularization, 1 patient died on POD 3 (patient 4), 1 patient went on to experience complete infarction of the contralateral ICA territory despite induced hypertension and required hemicraniectomy (patient 8), and 1 patient was deemed medically unstable for an additional revascularization procedure in the setting of postoperative gastrointestinal bleeding and ischemic colitis requiring laparotomy for bowel resection (patient 5) (Table 1).
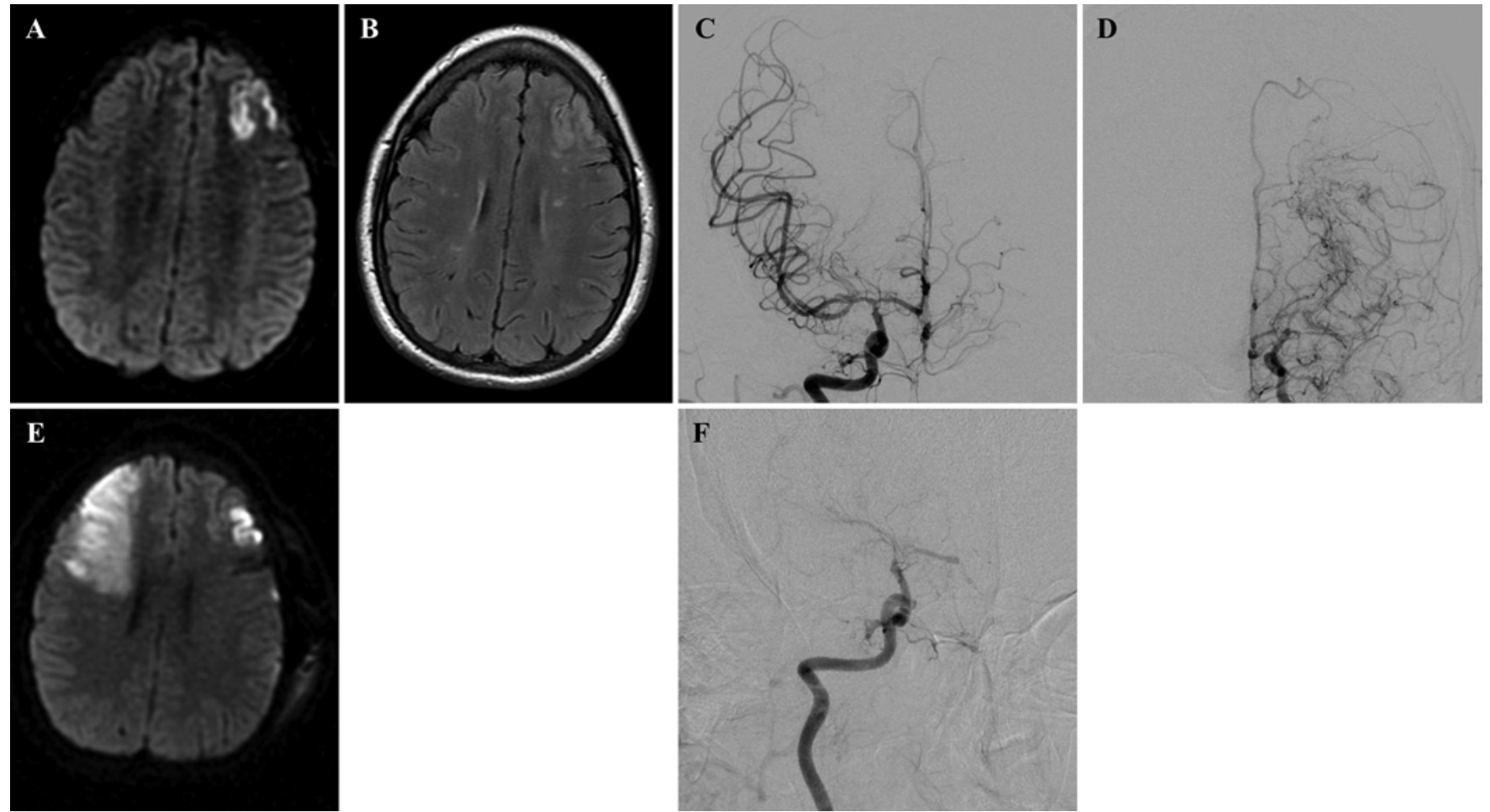

FIG. 1. Pre- and postoperative DSA and MR images for patient 1 in Table 1. Preoperative DWI (A) and FLAIR (B) MR images reveal an acute infarct in the left frontal lobe in an MCA watershed distribution, as well as evidence of chronic ischemic injury bilaterally in a watershed distribution. Preoperative DSA reveals moderate supraclinoid ICA stenosis on the right (C; contralateral to the bypass) and severe, high-grade preocclusive A1/M1 stenosis on the left (D; ipsilateral to the bypass). Postoperative MRI DWI sequence $(E)$ reveals new foci of acute infarct within the right frontal lobe extending into the right basal ganglia. Postoperative DSA (F) reveals progression to complete occlusion of the right (contralateral) supraclinoid ICA. 

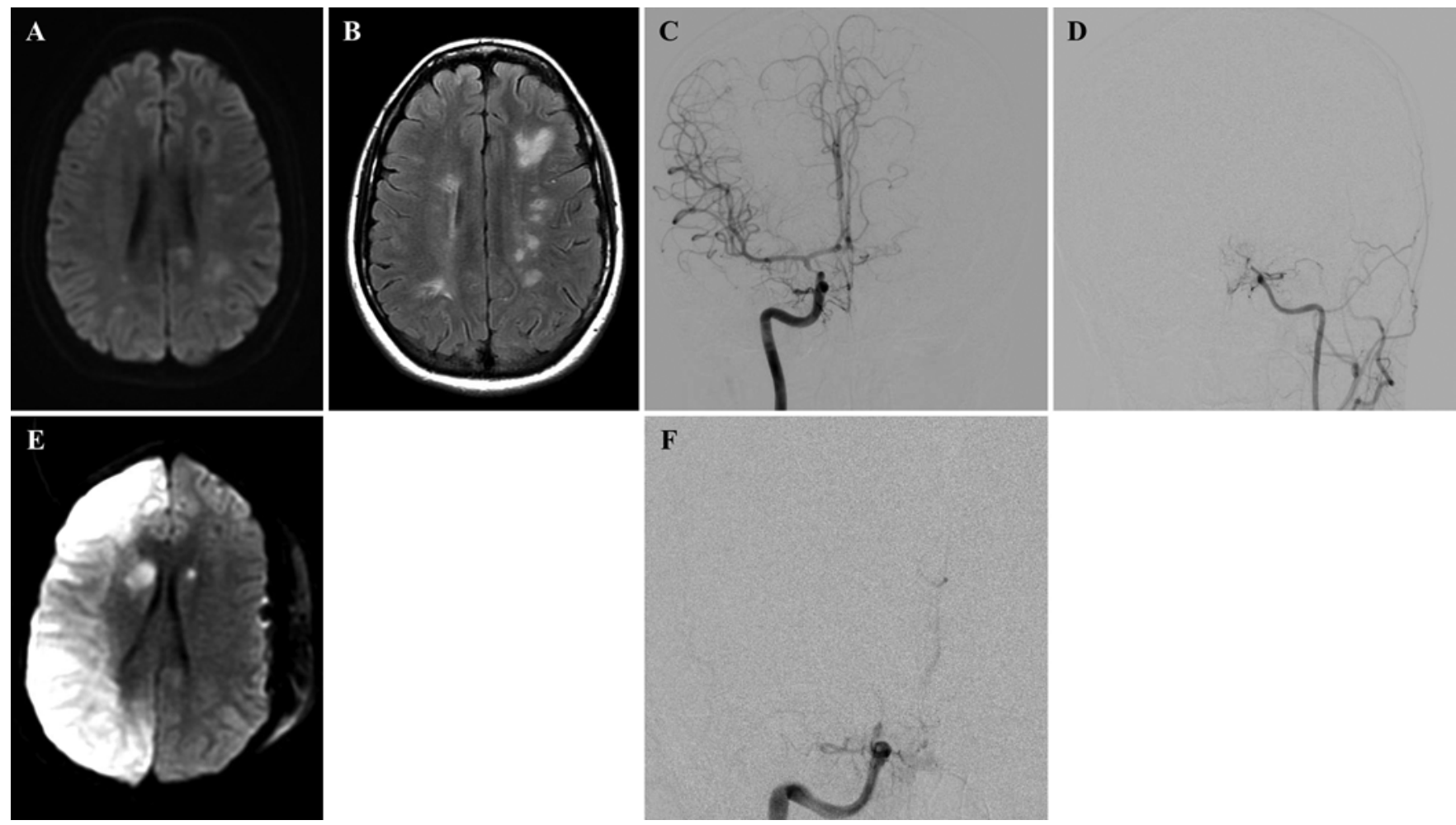

FIG. 2. Pre- and postoperative DSA and MR images for patient 4 in Table 1. Preoperative DWI (A) and FLAIR (B) MR images reveal evidence of bilateral chronic ischemic injury in a watershed distribution, with no evidence of acute infarct. Preoperative DSA reveals severe supraclinoid ICA stenosis on the right (C; contralateral to the bypass) and complete occlusion of the supraclinoid ICA on the left (D; ipsilateral to the bypass). Postoperative MRI DWI sequence (E) reveals new diffuse right holohemispheric cortical infarct with extension into the right caudate head and right thalamus, as well as a small infarct in the left caudate head. Postoperative DSA (F) reveals progression to complete occlusion of the right (contralateral) supraclinoid ICA.

The median length of stay for patients with acute contralateral ICA occlusion was 21 days (range 3-42 days). The median mRS score at discharge was 4 (range 1-6; data available for $8 / 8$ patients). At the 6-month follow-up, the median mRS score was 3 (range 1-6; data available for $7 / 8$ patients), and at the 3-year follow-up it was 2.5 (range 1-6; data available for 6/8 patients). The discharge $\mathrm{mRS}$ score was worse than the baseline mRS score in $7 / 8$ patients $(88 \%)$, whereas 1 patient had only minor deficits that did not affect the mRS score. At the 3-year follow-up, $3 / 8$ patients $(38 \%)$ were at their baseline mRS or better, 1 patient had significant disability compared with preoperatively, 2 patients had died, and 1 patient was lost to followup. One patient had not yet reached the 3-year follow-up at the time of this publication. Among the 3 patients who did not undergo contralateral revascularization during their index hospital course, 2 had died by the 6-month follow-up (patients 4 and 5), and 1 patient (patient 8) was dependent with significant disability compared with baseline (mRS score of 4 at 6 months vs mRS score of 1 at baseline) (Table 1).

\section{Discussion}

Surgical revascularization has been shown to decrease the risk of subsequent TIAs and strokes in patients with MMD; ;,4,7,17,19 however, this comes at the cost of increased upfront risk of stroke in the perioperative period. . $^{4,19}$ Well-designed studies have identified various risk factors for perioperative ischemic complications in revascularization surgery for MMD. In 1997, Sato et al. ${ }^{18}$ retrospectively reviewed the perioperative care of 36 patients who underwent revascularization surgery for MMD, 8 of whom experienced ischemic complications. The presence of a low-density area on preoperative CT, elevated intraoperative urinary output, and perioperative reduction of hematocrit were predictive factors for ischemic complications, while intraoperative hypercapnia, hypotension, and reduction in circulating blood volume were also identified as possible contributors..$^{18}$ In 2010 , Hyun et al.$^{9}$ retrospectively reviewed 246 revascularization procedures in 165 patients with MMD. In this multivariate regression analysis, the presence of a low-density area on preoperative CT or of DWI-positive lesions on preoperative MRI, history of $>4$ symptomatic ischemic episodes, and intraoperative hypercapnia, hypocapnia, hypotension, and hypovolemia were positively correlated with the risk of perioperative ischemic complications. ${ }^{9}$ In 2016, Antonucci et al. ${ }^{1}$ further demonstrated that an impaired cerebrovascular reserve and presence of a preoperative acute DWI-positive infarct, regardless of size or location, are independent predictors of severe postoperative ischemic complications following direct STA-MCA bypass for MMD.

Ischemic complications following revascularization 


\begin{tabular}{|c|c|c|c|c|c|c|c|c|c|c|c|c|c|c|c|}
\hline $\begin{array}{l}\text { Patient } \\
\text { No. }\end{array}$ & $\begin{array}{l}\text { Age } \\
\text { (yrs) }\end{array}$ & Sex & Race & $\begin{array}{l}\text { FLAIR } \\
\text { on } \\
\text { Preop } \\
\text { MRI }\end{array}$ & $\begin{array}{l}\text { DWI } \\
\text { on } \\
\text { Preop } \\
\text { MRI }\end{array}$ & $\begin{array}{c}\text { Degree } \\
\text { of Preop } \\
\text { Ipsilat } \\
\text { ICA } \\
\text { Stenosis }\end{array}$ & $\begin{array}{c}\text { Degree } \\
\text { of Preop } \\
\text { Contralat } \\
\text { ICA } \\
\text { Stenosis }\end{array}$ & $\begin{array}{c}\text { Collateral } \\
\text { Circulation } \\
\text { Pattern }\end{array}$ & $\begin{array}{l}\text { Suzuki } \\
\text { Grade }\end{array}$ & $\begin{array}{c}\text { Preop } \\
\text { mRS } \\
\text { Score }\end{array}$ & $\begin{array}{l}\text { LOS } \\
\text { (days) }\end{array}$ & $\begin{array}{c}\text { Discharge } \\
\text { mRS } \\
\text { Score }\end{array}$ & $\begin{array}{l}\text { 6-Mo } \\
\text { mRS } \\
\text { Score }\end{array}$ & $\begin{array}{l}3-Y r \\
m R S \\
\text { Score }\end{array}$ & $\begin{array}{c}\text { Contralat } \\
\text { Side } \\
\text { Bypass }\end{array}$ \\
\hline 1 & 34 & $\mathrm{~F}$ & Caucasian & + & + & Severe & Moderate & IC-IC & 4 & 1 & 30 & 4 & 3 & 3 & Yes \\
\hline 2 & 33 & $F$ & Asian & + & - & Severe & Moderate & IC-IC & 4 & 2 & 23 & 4 & 3 & 2 & Yes \\
\hline 3 & 51 & $F$ & Caucasian & + & - & Occluded & Moderate & EC-IC & 4 & 3 & 19 & 4 & NA & NA & Yes \\
\hline 4 & 30 & $\mathrm{~F}$ & Caucasian & + & - & Occluded & Severe & EC-IC & 5 & 2 & 3 & 6 & 6 & 6 & No \\
\hline 5 & 43 & $M$ & Caucasian & + & - & Occluded & Mild & EC-IC & 4 & 2 & 42 & 5 & 6 & 6 & No \\
\hline 6 & 49 & $\mathrm{~F}$ & Caucasian & - & - & Severe* & Mild* & IC-IC & 4 & 2 & 18 & 4 & 1 & 1 & Yes \\
\hline 7 & 22 & $F$ & Caucasian & + & - & Severe & Moderate & EC-IC & 4 & 1 & 16 & 1 & 1 & 1 & Yes \\
\hline 8 & 41 & $M$ & Caucasian & + & + & Occluded & Severe & IC-IC & 4 & 1 & 31 & 5 & 4 & NA & No \\
\hline
\end{tabular}

EC = extracranial; IC = intracranial; LOS = length of stay; NA = not applicable; + = present; - = absent.

* Preoperative DSA was obtained 99 days prior to the first surgical revascularization procedure.

surgery for MMD are not isolated to the ipsilateral hemisphere. In 2005, Kim et al. ${ }^{13}$ reported contralateral ischemic complications in $2.9 \%$ of revascularization surgeries for children. In 2011, Jung et al. ${ }^{11}$ retrospectively reviewed a series of adults with MMD and reported that $5.1 \%$ of these patients suffered from contralateral ischemic complications after surgical treatment for MMD; these authors further identified advanced Suzuki stage, posterior circulation involvement, and postoperative hypotension as risk factors for contralateral ischemic complications. In 2017, Kim et al. ${ }^{12}$ noted that contralateral (or bilateral) infarctions occurred more commonly following the first stage, compared with the second stage, of surgery in MMD patients undergoing bilateral revascularization surgery.

The precise mechanism by which the risk of stroke increases for MMD patients in the postoperative period is not clearly defined and is likely multifactorial. As described above, previous case series have described a phenomenon of rapid progression of ipsilateral intracranial stenoses following STA-MCA bypass for atherosclerotic arterial disease, including some patients who progress to complete occlusion during the immediate postoperative period..$^{2,5,6}$ The pathophysiological mechanism of this phenomenon has been hypothesized to involve changes in blood flow dynamics resulting in competing flows, with decreased velocity or reversal of blood flow across the stenotic lesion in the native vasculature. Others have attributed this phenomenon to a reduction in demand for blood flow via the native circulation, thereby resulting in slowed flow and subsequent stasis and occlusion. ${ }^{6,16}$

In this report, we described 8 cases of acute contralateral ICA occlusion following direct STA-MCA bypass surgery for MMD. This complication was uncommon, occurring in only $0.6 \%$ of cases in the senior author's series, but had a significant impact on the outcomes of affected patients. Our experience demonstrates that reasonable outcomes can be achieved despite this unexpected complication, if recognized early and managed aggressively.

The demographic and preoperative clinical characteristics of this patient cohort were representative of the entirety of our institution's MMD case series. In addition, no atypical intraoperative or postoperative hemodynamic factors were identified in affected patients. It is notable that 3 patients in this cohort underwent postoperative hematological workups, and an abnormality of hypercoagulability was found in all 3 patients (2 patients were heterozygous for factor $\mathrm{V}$ Leiden, and 1 patient tested positive for antiphospholipid syndrome). At the present time, hypercoagulability testing is not part of our institution's standard preoperative workup; nonetheless, this finding highlights the possibility that hematological factors may impact perioperative risk in MMD patients undergoing revascularization surgery. Also of note is the fact that all 8 patients experienced onset of symptoms during the immediate postoperative period; in most patients, symptoms began within just a few hours of surgery completion. Also of note is the responsiveness of symptoms to BP augmentation in $7 / 8$ patients. The fact that one patient did not demonstrate BP-dependent symptoms in the acute period, and that in another patient an infarct expanded and completed despite induced hypertension, may reflect inadequate collateral circulation. In 5 patients, aggressive supportive management and induced hypertension were maintained until the patient returned to the operating room for revascularization of the contralateral side; among this subgroup of 5 patients, 3 returned to their baseline functional status by the 3-year follow-up, and 1 patient had moderate disability but was independent at the 6-month follow-up (1 patient in this subgroup was lost to follow-up). No additional infarcts occurred in any of these patients following the second operation. In contrast, among the subgroup of patients who did not undergo contralateral revascularization, 1 patient died during the index hospitalization, 1 patient died prior to the 6-month follow-up, and 1 patient remained dependent at 6 months.

Given the dynamic nature of MMD, the standardized workup for revascularization surgery at our institution involves MRI, DSA, and cerebrovascular reserve studies during the immediate preoperative period. With the exception of 1 patient in the cohort reported here, preoperative DSA was obtained within 7 days of revascularization surgery. Upon recognition of new neurological deficits 
postoperatively, patients were evaluated with urgent MRI with perfusion imaging. Expedited DSA was subsequently obtained in selected patients. All 8 patients in this cohort were found to have significant contralateral perfusion delays on postoperative MRI, and DSA was subsequently obtained within 24 hours in each patient. While this protocol is standardized for acutely symptomatic patients in the postoperative period, it is important to note that not all patients in our MMD case series underwent routine postoperative imaging. Thus, it is likely that some perioperative infarcts, particularly those with minimal or no clinical significance, went unrecognized. In addition, DSA was not routinely obtained in the immediate postoperative period, raising the possibility that contralateral occlusion occurred in additional patients without a clinical correlate to prompt workup. It is also important to recognize that in the one patient who underwent preoperative DSA 3 months prior to revascularization surgery, we cannot rule out the possibility that the contralateral ICA progressed to complete occlusion prior to surgical intervention, as no immediate preoperative vascular imaging was obtained to establish disease stability.

It is well established that MMD is a dynamic and often relentlessly progressive pathologic process, and eventual progression of stenosis to complete occlusion may be part of the natural history of the disease. However, acute postoperative contralateral ICA occlusion with stroke after STA-MCA bypass has not been previously reported. This rare complication emphasizes the hemodynamic interrelationship and potential vascular collateral connections between the two hemispheres, a concept that has not been well described previously. The precise mechanism underlying acute contralateral ICA occlusion following STAMCA bypass is not clearly defined. We hypothesize that decreased flow velocity across an already stenotic vascular lumen results in further thrombosis and occlusion of the vessel. The decreased flow in the contralateral ICA following a direct bypass may be a result of decreased demand due to new blood supply via the direct bypass graft, or due to new retrograde blood flow as a result of altered cerebral blood flow dynamics - effectively, a phenomenon of "competing flows" between the graft and pre-bypass collaterals. The decrease in post-bypass global ipsilateral cerebral blood flow observed in some patients might support this concept of competing flows between graft and pre-bypass collaterals, and likewise competing flow from the graft to the contralateral hemisphere could promote occlusion of the stenotic ICA.

\section{Conclusions}

Acute perioperative occlusion of the contralateral ICA following STA-MCA bypass is a rare, but potentially serious, complication of revascularization surgery for MMD. This highlights the importance of the hemodynamic interrelationships between the two hemispheres, a previously underappreciated concept. Induced hypertension during the acute postoperative period may provide adequate cerebral blood flow via developing collateral vessels, and good outcomes may be achieved with aggressive supportive management and expedited contralateral revasculariza- tion. Future investigations should focus on determining the pathophysiological basis and predictors of this rare phenomenon.

\section{Acknowledgments}

We thank Christine Plant for editorial assistance.

\section{References}

1. Antonucci MU, Burns TC, Pulling TM, Rosenberg J, Marks MP, Steinberg GK, et al: Acute preoperative infarcts and poor cerebrovascular reserve are independent risk factors for severe ischemic complications following direct extracranialintracranial bypass for moyamoya disease. AJNR Am J Neuroradiol 37:228-235, 2016

2. Awad I, Furlan AJ, Little JR: Changes in intracranial stenotic lesions after extracranial-intracranial bypass surgery. J Neurosurg 60:771-776, 1984

3. Choi JU, Kim DS, Kim EY, Lee KC: Natural history of moyamoya disease: comparison of activity of daily living in surgery and non surgery groups. Clin Neurol Neurosurg 99 (Suppl 2):S11-S18, 1997

4. Fung LWE, Thompson D, Ganesan V: Revascularisation surgery for paediatric moyamoya: a review of the literature. Childs Nerv Syst 21:358-364, 2005

5. Furlan AJ, Little JR, Dohn DF: Arterial occlusion following anastomosis of the superficial temporal artery to middle cerebral artery. Stroke 11:91-95, 1980

6. Gumerlock MK, Ono H, Neuwelt EA: Can a patent extracranial-intracranial bypass provoke the conversion of an intracranial arterial stenosis to a symptomatic occlusion? Neurosurgery 12:391-400, 1983

7. Guzman R, Lee M, Achrol A, Bell-Stephens T, Kelly M, Do HM, et al: Clinical outcome after 450 revascularization procedures for moyamoya disease. Clinical article. J Neurosurg 111:927-935, 2009

8. Hallemeier CL, Rich KM, Grubb RL Jr, Chicoine MR, Moran CJ, Cross DT III, et al: Clinical features and outcome in North American adults with moyamoya phenomenon. Stroke 37:1490-1496, 2006

9. Hyun SJ, Kim JS, Hong SC: Prognostic factors associated with perioperative ischemic complications in adult-onset moyamoya disease. Acta Neurochir (Wien) 152:1181-1188, 2010

10. Imaizumi T, Hayashi K, Saito K, Osawa M, Fukuyama Y: Long-term outcomes of pediatric moyamoya disease monitored to adulthood. Pediatr Neurol 18:321-325, 1998

11. Jung YJ, Ahn JS, Kwon DH, Kwun BD: Ischemic complications occurring in the contralateral hemisphere after surgical treatment of adults with moyamoya disease. J Korean Neurosurg Soc 50:492-496, 2011

12. Kim HG, Lee SK, Lee JD: Characteristics of infarction after encephaloduroarteriosynangiosis in young patients with moyamoya disease. J Neurosurg Pediatr 19:1-7, 2017

13. Kim SH, Choi JU, Yang KH, Kim TG, Kim DS: Risk factors for postoperative ischemic complications in patients with moyamoya disease. J Neurosurg 103 (5 Suppl):433-438, 2005

14. Kuroda S, Ishikawa T, Houkin K, Nanba R, Hokari M, Iwasaki Y: Incidence and clinical features of disease progression in adult moyamoya disease. Stroke 36:2148-2153, 2005

15. Kurokawa T, Chen YJ, Tomita S, Kishikawa T, Kitamura $\mathrm{K}$ : Cerebrovascular occlusive disease with and without the moyamoya vascular network in children. Neuropediatrics 16:29-32, 1985

16. Liu JJ, Steinberg GK: Direct versus indirect bypass for moyamoya disease. Neurosurg Clin N Am 28:361-374, 2017

17. Nakashima H, Meguro T, Kawada S, Hirotsune N, Ohmoto 
T: Long-term results of surgically treated moyamoya disease. Clin Neurol Neurosurg 99 (Suppl 2):S156-S161, 1997

18. Sato K, Shirane R, Yoshimoto T: Perioperative factors related to the development of ischemic complications in patients with moyamoya disease. Childs Nerv Syst 13:68-72, 1997

19. Scott RM, Smith JL, Robertson RL, Madsen JR, Soriano SG, Rockoff MA: Long-term outcome in children with moyamoya syndrome after cranial revascularization by pial synangiosis. J Neurosurg 100 (2 Suppl Pediatrics):142-149, 2004

20. Suzuki J, Takaku A: Cerebrovascular "moyamoya" disease. Disease showing abnormal net-like vessels in base of brain. Arch Neurol 20:288-299, 1969

21. Takeuchi K, Shimizu K: Hypoplasia of the bilateral internal carotid arteries. Brain Nerve 9:37-43, 1957

\section{Disclosures}

This study was supported in part by Russell and Elizabeth Siegelman, Bernard and Ronni Lacroute, and the William Randolph Hearst Foundation (G.K.S.).

Dr. Steinberg is a consultant for Qool Therapeutics, Peter Lazic US, Inc., and NeuroSave.

\section{Author Contributions}

Conception and design: Steinberg, Madhugiri, Teo, Furtado. Acquisition of data: Sussman, Madhugiri, Teo, Furtado, Esparza, Azad, Zhang. Analysis and interpretation of data: Steinberg, Sussman, Teo, Nielsen, Furtado, Pendharkar. Drafting the article: Sussman, Nielsen, Furtado, Pendharkar, Ho. Critically revising the article: Steinberg, Sussman, Madhugiri, Teo, Nielsen, Pendharkar, Ho. Reviewed submitted version of manuscript: Steinberg, Sussman, Madhugiri, Teo, Nielsen, Pendharkar, Ho, Esparza, Azad, Zhang. Approved the final version of the manuscript on behalf of all authors: Steinberg. Statistical analysis: Sussman, Furtado. Study supervision: Steinberg.

\section{Correspondence}

Gary K. Steinberg: Stanford University School of Medicine, Stanford, CA. gsteinberg@stanford.edu. 